\title{
The Time Delays' Effects on the Qualitative Behavior of an Economic Growth Model
}

\author{
Carlo Bianca, ${ }^{1}$ Massimiliano Ferrara, ${ }^{2}$ and Luca Guerrini ${ }^{3}$ \\ ${ }^{1}$ Dipartimento di Scienze Matematiche, Politecnico, Corso Duca degli Abruzzi 24, 10129 Torino, Italy \\ ${ }^{2}$ Department of Law and Economics, University Mediterranea of Reggio Calabria and CRIOS University Bocconi of Milan, \\ Via dei Bianchi 2, 89127 Reggio Calabria, Italy \\ ${ }^{3}$ Department of Management, Polytechnic University of Marche, 60121 Ancona, Italy
}

Correspondence should be addressed to Carlo Bianca; carlo.bianca@polito.it

Received 14 August 2013; Accepted 1 November 2013

Academic Editor: Constantin Udriste

Copyright (c) 2013 Carlo Bianca et al. This is an open access article distributed under the Creative Commons Attribution License, which permits unrestricted use, distribution, and reproduction in any medium, provided the original work is properly cited.

\begin{abstract}
A further generalization of an economic growth model is the main topic of this paper. The paper specifically analyzes the effects on the asymptotic dynamics of the Solow model when two time delays are inserted: the time employed in order that the capital is used for production and the necessary time so that the capital is depreciated. The existence of a unique nontrivial positive steady state of the generalized model is proved and sufficient conditions for the asymptotic stability are established. Moreover, the existence of a Hopf bifurcation is proved and, by using the normal form theory and center manifold argument, the explicit formulas which determine the stability, direction, and period of bifurcating periodic solutions are obtained. Finally, numerical simulations are performed for supporting the analytical results.
\end{abstract}

\section{Introduction}

Most of the phenomena occurring in real-world complex systems, especially in the economics systems, have not an immediate effect but appear with some delay. Therefore time delays have been inserted into mathematical models and in particular in models of the applied sciences based on ordinary differential equations; see the recent book [1]. Differential equations specifically with time delays have been proposed in population dynamics [2] for biological systems such as immune system response [3-6] and tumor growth [7-12], in models of social sciences [13], and in economics systems; see, among others, [14-21].

The introduction of a time delay into an ordinary differential equation could change the stability of the equilibrium (stable equilibrium becomes unstable) and could cause fluctuations, and Hopf bifurcation can occur. Indeed global existence of Hopf bifurcations has been proved in many delay mathematical models; see papers [22-24] and references cited therein.

If on one hand, the stability and bifurcation analysis of ordinary differential equations with a single time delay is well outlined in the pertinent literature $[25,26]$, on the other hand the analysis of the dynamics of ordinary differential equations with multiple time delays is a difficult task [27-29] and the related literature is much limited. In this context, for several classes of ordinary differential equation models with multiple time delays, sufficient and necessary conditions have been established and a complete description of the stability region has been reached; see, among others, $[30,31]$ and references cited therein.

The present paper is concerned with a further generalization of the Solow model [32]. The generalized model is governed by a delay differential equation with two time delays. Specifically the two time delays refer, respectively, to the time employed in order that the capital is used for production and the necessary time so that the capital is depreciated. The asymptotic analysis performed in this paper shows the existence of a unique nontrivial positive steady state, and sufficient conditions for the asymptotic stability are established. Moreover, the existence of a Hopf bifurcation is proved and, by using the normal form theory and center manifold argument, the explicit formulas which determine the stability, direction, and period of bifurcating periodic solutions are 
obtained. Finally, some numerical simulations to support the analytical conclusions are carried out.

The rest of the paper is organized into four more sections which follow this introduction. Specifically, Section 2 discusses the derivation of the generalized Solow model with two time delays. Section 3 deals with the analysis of the existence of Hopf bifurcation and stability of the positive equilibrium for the model proposed in Section 1. Section 4 is concerned with the direction and the stability of the Hopf bifurcation. Some numerical simulations are performed in Section 5 with the aim of supporting the analytic results. Finally Section 6 completes the paper with conclusions and some future research perspectives.

\section{The Mathematical Model}

Recently, Zak has proposed in [15] the following delay ordinary differential equation

$$
\dot{k}(t)=s f(k(t-\tau))-\delta k(t-\tau)
$$

to describe the dynamics of the Solow model [32] in which a production technology has a constant finite period $\tau \geq$ 0 linked to the time needed for the installation of capital. Here, $k(t)$ denotes capital at time $t, f(k(t))$ is a neoclassical production function, namely a function which is continuous, increasing, and strictly concave in capital, and $s \in(0,1)$ is the constant savings rate. Population is assumed to be constant and normalized to unity. During production a proportion of the capital stock, $\delta \in(0,1)$, depreciates at the same gestation period $\tau$.

The assumption that the growth of the amount of capital at time $t$ is a function of the total output of capital at time $t-\tau$ has been showed in [15] to be the source of cyclic behaviour in the economic system (1). Generalizing this idea, we proposed the following delay ordinary differential equation:

$$
\dot{k}(t)=s f\left(k\left(t-\tau_{1}\right)\right)-\delta k\left(t-\tau_{2}\right),
$$

that is, the generalized Solow model with two delays.

It is worth stressing that for $\tau_{1}=\tau_{2}=\tau=0$, we recover the Solow model equation [32]. In this model, the positive equilibrium is asymptotically stable in the absence of delay. For $\tau_{1}=\tau_{2}=\tau>0,(2)$ reduces to the delayed Solow model proposed by Zak in [15].

\section{Local Stability and Hopf Bifurcation}

The mathematical model (2) has exactly the same equilibrium points of the corresponding system with zero delays. Hence, there exists a unique positive equilibrium $k_{*}$, where $s f\left(k_{*}\right)=$ $\delta k_{*}$. To determine the stability of this equilibrium and Hopf bifurcation, we linearize (2) around $k_{*}$. The result is a linear delay differential equation of the form

$$
\dot{k}(t)=-A_{1}\left[k\left(t-\tau_{1}\right)-k_{*}\right]-A_{2}\left[k\left(t-\tau_{2}\right)-k_{*}\right],
$$

where

$$
A_{1}=\left[s f^{\prime}\left(k_{*}\right)-\delta\right]<0, \quad A_{2}=\delta>0 .
$$

It is well known that the stability of the equilibrium is determined by the spectrum of the eigenvalues of the linearization, which can be found as the roots of the characteristic equation

$$
\lambda=-A_{1} e^{-\lambda \tau_{1}}-A_{2} e^{-\lambda \tau_{2}} .
$$

We recall that an equilibrium point of an equation is stable if all eigenvalues of its linearization have negative real part. It changes its stability type when eigenvalues cross the imaginary axis of the complex plane. We first note that $\lambda=0$ is not a root of (5) because this would imply $s f^{\prime}\left(k_{*}\right)=0$, contradicting the fact that $f^{\prime}\left(k_{*}\right)>0$. Next, the distribution of the roots of (5) should be investigated. However, the analysis of the sign of the real parts of eigenvalues is very complicated because of the presence of two different delays, $\lambda_{1}$ and $\lambda_{2}$, in (5). Therefore, we will use a method consisting of determining the stability of the equilibrium when one delay is equal to zero, and, using similar analytic arguments as in the work by Ruan and Wei [33], we will deduce conditions for the stability of the equilibrium when both time delays are nonzero.

3.1. Case 1: $\tau_{1} \geq 0$ and $\tau_{2}=0$. The characteristic equation (5) reduces to

$$
\lambda=-A_{1} e^{-\lambda \tau_{1}}-A_{2}
$$

If $\tau_{1}=0$, (6) has the unique root $\lambda=-A_{1}-A_{2}=-s f^{\prime}\left(k_{*}\right)<$ 0 . Thus, the equilibrium $k_{*}$ is locally asymptotically stable. Consequently, when $\tau_{1}$ increases, the stability of the steady state can only be lost if pure imaginary roots appear. Hence we look for purely imaginary roots $\pm i \omega, \omega>0$, of (6). Let $i \omega$ be a purely imaginary root of (6). Then, separating real and imaginary parts, $\omega$ satisfies

$$
\omega=A_{1} \sin \left(\omega \tau_{1}\right), \quad A_{2}=-A_{1} \cos \left(\omega \tau_{1}\right) .
$$

It follows that

$$
\begin{aligned}
\omega^{2} & =A_{1}^{2}-A_{2}^{2}=\left(A_{1}+A_{2}\right)\left(A_{1}-A_{2}\right) \\
& =s f^{\prime}\left(k_{*}\right)\left\{\left[s f^{\prime}\left(k_{*}\right)-\delta\right]-\delta\right\}<0 .
\end{aligned}
$$

Hence, (6) has no positive root. Then, we can conclude the following result about the asymptotic stability of the equilibrium of (2).

Proposition 1. Let $\tau_{2}=0$. Then the positive equilibrium $k_{*}$ of (2) is locally asymptotically stable.

3.2. Case 2: $\tau_{1}=0$ and $\tau_{2} \geq 0$. The characteristic equation (5) becomes

$$
\lambda=-A_{1}-A_{2} e^{-\lambda \tau_{2}}
$$

Setting $\tau_{2}=0$, we know that the equilibrium $k_{*}$ is locally asymptotically stable. Let $\lambda=i \omega, \omega>0$, be a root of (9). Separating real and imaginary parts, we have the following two equations:

$$
\omega=A_{2} \sin \left(\omega \tau_{2}\right), \quad A_{1}=A_{2} \cos \left(\omega \tau_{2}\right) .
$$


Adding the squares of both hand sides of (10), it follows that $\omega$ must be a root of the following equation:

$$
\begin{aligned}
\omega^{2} & =A_{2}^{2}-A_{1}^{2}=\left(-A_{1}-A_{2}\right)\left(A_{1}-A_{2}\right) \\
& =-s f^{\prime}\left(k_{*}\right)\left\{\left[s f^{\prime}\left(k_{*}\right)-\delta\right]-\delta\right\}>0 .
\end{aligned}
$$

Hence, (11) admits only the positive root

$$
\omega_{0}=\sqrt{A_{2}^{2}-A_{1}^{2}} .
$$

Define from (10)

$$
\tau_{2}^{0}=\frac{1}{\omega_{0}} \arccos \left(\frac{A_{1}}{A_{2}}\right)
$$

and

$$
\tau_{2}^{n}=\tau_{2}^{0}+\frac{2 n \pi}{\omega_{0}}, \quad n=0,1,2, \ldots
$$

Then, it is immediate to check that $i \omega_{0}$ is a simple root of (9) when $\tau_{2}=\tau_{2}^{n}$.

Lemma 2. Let $\lambda\left(\tau_{2}\right)=a\left(\tau_{2}\right)+i \omega\left(\tau_{2}\right)$ be the complex root of (9) near $\tau_{2}=\tau_{2}^{n}$ satisfying $a\left(\tau_{2}^{n}\right)=0$ and $\omega\left(\tau_{2}^{n}\right)=\omega_{0}$. Then the transversal condition

$$
\operatorname{sign}\left\{\frac{d \operatorname{Re}\left[\lambda\left(\tau_{2}^{n}\right)\right]}{d \tau_{2}}\right\}>0
$$

is satisfied.

Proof. Differentiating the characteristic equation (9) with respect to $\tau_{2}$, we obtain

$$
\frac{d \lambda}{d \tau_{2}}=A_{2} e^{-\tau_{2} \lambda}\left(\tau_{2} \frac{d \lambda}{d \tau_{2}}+\lambda\right)
$$

This gives

$$
\left(\frac{d \lambda}{d \tau_{2}}\right)^{-1}=-\frac{1}{\lambda\left(\lambda+A_{1}\right)}-\frac{\tau_{2}}{\lambda} .
$$

Hence, we have

$$
\begin{aligned}
\operatorname{sign}\left[\frac{d(\operatorname{Re} \lambda)}{d \tau_{2}}\right]_{\lambda=i \omega_{0}, \tau_{2}=\tau_{2}^{n}} & =\operatorname{sign}\left[\operatorname{Re}\left(\frac{d \lambda}{d \tau_{2}}\right)_{\lambda=i \omega_{0}, \tau_{2}=\tau_{2}^{n}}^{-1}\right] \\
& =\operatorname{sign}\left[\frac{1}{\omega_{0}^{2}+A_{1}^{2}}\right] .
\end{aligned}
$$

This completes the proof.

Bearing the above analysis in mind and the Hopf bifurcation theorem for functional differential equations due to Hale and Verduyn Lunel (see p. 246, Theorem 1.1 of the book [34]), we have the following result.

Theorem 3. Let $\tau_{1}=0$. Then the positive equilibrium $k_{*}$ of (2) is locally asymptotically stable when $\tau_{2} \in\left[0, \tau_{2}^{0}\right)$ and unstable when $\tau_{2}>\tau_{2}^{0}$. Furthermore, (2) undergoes a Hopf bifurcation at the positive equilibrium $k_{*}$ when $\tau_{2}=\tau_{2}^{n}, n=0,1,2, \ldots$, where $\tau_{2}^{n}$ is defined as in (14).
3.3. Case 3: $\tau_{1}>0$ and $\tau_{2}>0$. Equation (5) has purely imaginary roots $\pm i \omega$, where $\omega>0$, if the following equations are satisfied. Consider

$$
\begin{gathered}
\omega-A_{1} \sin \left(\omega \tau_{1}\right)=A_{2} \sin \left(\omega \tau_{2}\right), \\
A_{1} \cos \left(\omega \tau_{1}\right)=-A_{2} \cos \left(\omega \tau_{2}\right) .
\end{gathered}
$$

Squaring and adding up both equations in (19) yield

$$
\sin \left(\omega \tau_{1}\right)=g(\omega),
$$

where

$$
g(\omega)=\frac{\omega^{2}+A_{1}^{2}-A_{2}^{2}}{2 \omega A_{1}}
$$

Lemma 4. For every arbitrary $\tau_{1}>0,(20)$ has a finite number of positive solutions for $\omega \in\left[A_{1}+A_{2},-A_{1}+A_{2}\right]$.

Proof. The inequality $\left|\sin \left(\omega \tau_{1}\right)\right| \leq 1$ and $A>1$ imply $\omega \in$ $\left[A_{1}+A_{2},-A_{1}+A_{2}\right]$. The function $g(\omega)$ has the properties $g\left(A_{1}+A_{2}\right)=1, g\left(-A_{1}+A_{2}\right)=-1, g\left(\sqrt{A_{2}^{2}-A_{1}^{2}}\right)=0$, and

$$
\frac{d g(\omega)}{d \omega}=\frac{\omega^{2}+A_{2}^{2}-A_{1}^{2}}{2 \omega^{2} A_{1}}<0, \quad \frac{d^{2} g(\omega)}{d \omega^{2}}=\frac{A_{1}^{2}-A_{2}^{2}}{\omega^{3} A_{1}^{2}}<0 .
$$

A graphical inspection on the intersections of the functions $\sin \left(\omega \tau_{1}\right)$ and $g(\omega)$ gives the statement.

Remark 5. If $\tau_{1} \in\left(0, \pi /\left(-A_{1}+A_{2}\right)\right)$, (20) has only one positive solution.

For any $\tau_{1}>0,(20)$ has a finite number of positive zeros $\omega_{j}, j=1,2, \ldots, m$. It is clear that for every arbitrary chosen $\tau_{1}>0$ and for each $\omega_{j}$ we have an infinite number of $\tau_{2}$ such that $A_{1} \cos \left(\omega_{j} \tau_{1}\right)=-A_{2} \cos \left(\omega_{j} \tau_{2}\right)$. For all $j=1,2, \ldots, m$, we define

$$
\tau_{2}^{j}=\min \left\{\tau_{2}>0: A_{1} \cos \left(\omega_{j} \tau_{1}\right)=-A_{2} \cos \left(\omega_{j} \tau_{2}\right)\right\} .
$$

In addition, we set

$$
\tau_{2}^{0}=\min \left\{\tau_{2}^{j}: j=1,2, \ldots, m\right\}
$$

and denote $\omega_{0}=\omega_{j}$ for $j$ such that $\tau_{2}^{j}=\tau_{2}^{0}$. Next, we check the condition which guarantees that the purely imaginary roots pass through the imaginary axis at $\pm i \omega_{0}$. Let $\lambda\left(\tau_{2}\right)=a\left(\tau_{2}\right)+$ $i \omega\left(\tau_{2}\right)$ be the root of $(5)$ near $\tau_{2}=\tau_{2}^{0}$ such that $a\left(\tau_{2}^{0}\right)=0$ and $\omega\left(\tau_{2}^{0}\right)=\omega_{0}$. By direct computation we have

$$
\frac{d \lambda}{d \tau_{2}}=A_{1} e^{-\lambda \tau_{1}}\left(\tau_{1} \frac{d \lambda}{d \tau_{2}}\right)+A_{2} e^{-\lambda \tau_{2}}\left(\tau_{2} \frac{d \lambda}{d \tau_{2}}+\lambda\right)
$$

and obtain

$$
\left(\frac{d \lambda}{d \tau_{2}}\right)^{-1}=-\frac{1+\lambda \tau_{1}}{\lambda A_{2} e^{-\lambda \tau_{2}}}+\frac{\tau_{1}-\tau_{2}}{\lambda} .
$$


Hence, it follows that

$$
\begin{aligned}
& \operatorname{sign}\left[\frac{d(\operatorname{Re} \lambda)}{d \tau_{2}}\right]_{\lambda=i \omega_{0}, \tau_{2}=\tau_{2}^{0}} \\
& =\operatorname{sign}\left[\operatorname{Re}\left(\frac{d \lambda}{d \tau_{2}}\right)_{\lambda=i \omega_{0}, \tau_{2}=\tau_{2}^{0}}^{-1}\right] \\
& =\operatorname{sign}\left[\frac{\sin \left(\omega_{0} \tau_{2}^{0}\right)+\tau_{1} \omega_{0} \cos \left(\omega_{0} \tau_{2}^{0}\right)}{A_{2} \omega_{0}}\right] \\
& =\operatorname{sign}\left[\sin \left(\omega_{0} \tau_{2}^{0}\right)+\tau_{1} \omega_{0} \cos \left(\omega_{0} \tau_{2}^{0}\right)\right] .
\end{aligned}
$$

If for given $\tau_{1}$ both $\sin \left(\omega_{0} \tau_{2}^{0}\right)$ and $\cos \left(\omega_{0} \tau_{2}^{0}\right)$ are positive (resp., negative), then

$$
\operatorname{sign}\left[\sin \left(\omega_{0} \tau_{2}^{0}\right)+\tau_{1} \omega_{0} \cos \left(\omega_{0} \tau_{2}^{0}\right)\right]>0 \quad(\text { resp., }<0)
$$

and the purely imaginary roots $\pm i \omega_{0}$ of (5) move to the right (resp., left) half plane when the bifurcation parameter $\tau_{2}$ increases. So, we have the following transversality condition.

Proposition 6. Let $\Omega_{1}=[0, \pi / 2]$ and $\Omega_{2}=[\pi, 3 \pi / 2]$.

(1) If $\omega_{0} \tau_{2}^{0} \in \Omega_{1}$, then sign $\left[d(\operatorname{Re} \lambda) / d \tau_{2}\right]_{\lambda=i \omega_{0}, \tau_{2}=\tau_{2}^{0}}>0$.

(2) If $\omega_{0} \tau_{2}^{0} \in \Omega_{2}$, then sign $\left[d(\operatorname{Re} \lambda) / d \tau_{2}\right]_{\lambda=i \omega_{0}, \tau_{2}=\tau_{2}^{0}}<0$.

Lemma 7. $\lambda= \pm i \omega_{0}$ are simple roots of (5) when $\omega_{0} \tau_{2}^{0} \in \Omega_{1} \cup$ $\Omega_{2}$.

Proof. If $\lambda=i \omega_{0}$ is a repeated root for (5), then $1=$ $\tau_{1} A_{1} e^{-i \omega_{0} \tau_{1}}+\tau_{2}^{0} A_{2} e^{-i \omega_{0} \tau_{2}^{0}}$ holds true. Using (5), this leads to $-\omega_{0} \tau_{1}=\left(\tau_{2}^{0}-\tau_{1}\right) A_{2} \sin \left(\omega_{0} \tau_{2}^{0}\right)$ and $1=\left(\tau_{2}^{0}-\tau_{1}\right) A_{2} \cos \left(\omega_{0} \tau_{2}^{0}\right)$. Thus, we must have $-\omega_{0} \tau_{1}=\tan \left(\omega_{0} \tau_{2}^{0}\right)$. If $\omega_{0} \tau_{2}^{0} \in \Omega_{1}$, this means $\omega_{0}=0$, while if $\omega_{0} \tau_{2}^{0} \in \Omega_{2}$, this identity does not hold. The conclusion is immediate.

From the discussion above, and recalling that for any $\tau_{1}$ and $\tau_{2}=0$ all roots of (5) have strictly negative real parts, the following theorem about stability and Hopf bifurcation of (2) is immediately obtained.

Theorem 8. Let $\tau_{1}>0$ and $\Omega_{1}, \Omega_{2}$ be defined as in Proposition 6. Then

(1) equation (2) undergoes a Hopf bifurcation at $k_{*}$ when $\tau_{2}=\tau_{2}^{0}$,

(2) if $\omega_{0} \tau_{2}^{0} \in \Omega_{1}$, then the nontrivial equilibrium $k_{*}$ to (2) is locally asymptotically stable for $\tau_{2} \in\left[0, \tau_{2}^{0}\right)$ and unstable for $\tau_{2}>\tau_{2}^{0}$,

(3) if $\omega_{0} \tau_{2}^{0} \in \Omega_{2}$, then the nontrivial equilibrium $k_{*}$ to (2) is locally asymptotically stable for $\tau_{2} \in[0,+\infty)$.

\section{Direction and Stability of the Hopf Bifurcation}

In this section, we study the direction of bifurcations and the stability of bifurcating periodic solutions of (2) at $\tau_{2}^{0}$ by using the method based on the normal form theory and center manifold theory introduced by Hassard et al. [35].

For notational convenience, let $\tau_{2}=\tau_{2}^{0}+\mu, \mu \in \mathbb{R}$. Then $\mu=0$ is the Hopf bifurcation point for (2). First, we transform (2) into a functional differential equation in $C\left(\left[-\tau_{1}, 0\right], \mathbb{R}\right)$, which is the Banach space of continuous real-valued functions that map $\left[-\tau_{1}, 0\right]$ into $\mathbb{R}$, and endowed with the norm

$$
\|\varphi\|=\sup _{-\tau_{1} \leq \vartheta \leq 0}|\varphi(\vartheta)| .
$$

Set $x(t)=k(t)-k_{*}$. Then, rewriting (2) in terms of $x(t)$ and considering its Taylor expansion at the trivial equilibrium up to the third order, we get

$$
\begin{aligned}
\dot{x}(t)= & -\left[s f^{\prime}\left(k_{*}\right)-\delta\right] x\left(t-\tau_{1}\right)-\delta x\left(t-\tau_{2}\right) \\
& +\frac{s f^{\prime \prime}\left(k_{*}\right)}{2} x\left(t-\tau_{1}\right)^{2}+\frac{s f^{\prime \prime \prime}\left(k_{*}\right)}{3 !} x\left(t-\tau_{1}\right)^{3}+\cdots .
\end{aligned}
$$

For $\varphi \in C\left(\left[-\tau_{1}, 0\right], \mathbb{R}\right)$, define the linear operator

$$
L_{\mu}(\varphi)=-\left[s f^{\prime}\left(k_{*}\right)-\delta\right] \varphi\left(-\tau_{1}\right)-\delta \varphi\left(-\tau_{2}\right)
$$

and the nonlinear operator

$$
F(\mu, \varphi)=\frac{s f^{\prime \prime}\left(k_{*}\right)}{2} \varphi\left(-\tau_{1}\right)^{2}+\frac{s f^{\prime \prime \prime}\left(k_{*}\right)}{3 !} \varphi\left(-\tau_{1}\right)^{3}+\cdots .
$$

By the Riesz representation theorem, there exists a bounded variation function $\eta(\vartheta, \mu)$ with $\vartheta \in\left[-\tau_{1}, 0\right]$ such that

$$
L_{\mu}(\varphi)=\int_{-\tau_{1}}^{0} d \eta(\vartheta, \mu) \varphi(\vartheta)
$$

with

$$
\eta(\vartheta, \mu)= \begin{cases}-\delta \Gamma(\vartheta), & \vartheta \in\left(-\tau_{2}, 0\right], \\ {\left[s f^{\prime}\left(k_{*}\right)-\delta\right] \Gamma\left(\vartheta+\tau_{1}\right),} & \vartheta \in\left[-\tau_{1},-\tau_{2}\right],\end{cases}
$$

where $\Gamma$ is the Dirac delta function. For $\varphi \in C\left(\left[-\tau_{1}, 0\right], \mathbb{R}\right)$, define

$$
\begin{aligned}
& A(\mu) \varphi= \begin{cases}\frac{d \varphi(\vartheta)}{d \vartheta}, & \vartheta \in\left[-\tau_{1}, 0\right), \\
\int_{-\tau_{1}}^{0} d \eta(u, \mu) \varphi(u), & \vartheta=0,\end{cases} \\
& R(\mu) \varphi= \begin{cases}0, & \vartheta \in\left[-\tau_{1}, 0\right), \\
F(\mu, \varphi), & \vartheta=0 .\end{cases}
\end{aligned}
$$

Then (30) is equivalent to

$$
\dot{x}_{t}=A(\mu) x_{t}+R(\mu) x_{t},
$$

where $x_{t}(\vartheta)=x(t+\vartheta)$ for $\vartheta \in\left[-\tau_{1}, 0\right]$. For $\psi \in C\left(\left[0, \tau_{1}\right], \mathbb{R}\right)$, define the operator $A^{*}$ as

$$
A^{*}(0) \psi(u)= \begin{cases}-\frac{d \psi(u)}{d u}, & u \in\left(0, \tau_{1}\right], \\ \int_{-\tau_{1}}^{0} d \eta(r, 0) \psi(-r), & u=0,\end{cases}
$$


and the bilinear inner product

$$
\begin{aligned}
\langle\psi, \varphi\rangle= & \bar{\psi}(0) \varphi(0) \\
& -\int_{\vartheta=-\tau_{1}}^{0} \int_{\xi=0}^{\vartheta} \bar{\psi}(\xi-\vartheta) d \eta(\vartheta, 0) \varphi(\xi) d \xi,
\end{aligned}
$$

where the over bar denotes complex conjugation. Then $A(0)$ and $A^{*}(0)$ are adjoint operators. By the discussion in the previous section, we know that $\pm i \omega_{0}$ are eigenvalues of $A(0)$. Thus, they are also eigenvalues of $A^{*}(0)$. We need to compute the eigenvector of $A(0)$ and $A^{*}(0)$ corresponding to $i \omega_{0}$ and $-i \omega_{0}$, respectively. A direct computation shows that their eigenvectors are

$$
\begin{gathered}
q(\vartheta)=e^{i \omega_{0} \vartheta}, \quad \vartheta \in\left[-\tau_{1}, 0\right], \\
q^{*}(u)=B e^{i \omega_{0} u}, \quad u \in\left[0, \tau_{1}\right],
\end{gathered}
$$

respectively. We have $\left\langle q^{*}, \bar{q}\right\rangle=0$. In order to ensure $\left\langle q^{*}, q\right\rangle=$ 1 , we choose $B$ as

$$
B=\frac{1}{1-\tau_{1}\left[s f^{\prime}\left(k_{*}\right)-\delta\right] e^{i \omega_{0} \tau_{1}}-\tau_{2}^{0} \delta e^{i \omega_{0} \tau_{2}^{0}}} .
$$

Next, we compute the coordinates to describe the center manifold $\mathscr{C}$ at $\mu=0$. Let $x_{t}$ be the solution of (36) when $\mu=0$. Define

$$
z(t)=\left\langle q^{*}, x_{t}\right\rangle, \quad W(t, \vartheta)=x_{t}(\vartheta)-2 \operatorname{Re}[z(t) q(\vartheta)] .
$$

On the center manifold $\mathscr{C}$, we have $W(t, \vartheta)=W(z(t), \bar{z}(t), \vartheta)$, with

$$
W(z, \bar{z}, \vartheta)=W_{20}(\vartheta) \frac{z^{2}}{2}+W_{11}(\vartheta) z \bar{z}+W_{02}(\vartheta) \frac{\bar{z}^{2}}{2}+\cdots
$$

where $z$ and $\bar{z}$ are local coordinates for $\mathscr{C}$ in the direction of $q^{*}$ and $\bar{q}^{*}$. For any $x_{t} \in \mathscr{C}$, since $\mu=0$, we find

$$
\dot{z}(t)=i \omega_{0} z(t)+\bar{q}^{*}(0) F_{0}(z, \bar{z}),
$$

with

$$
F_{0}(z, \bar{z})=F(0, W(z, \bar{z}, 0)+2 \operatorname{Re}[z(t) q(0)]) .
$$

We rewrite (43) as

$$
\dot{z}(t)=i \omega_{0} z(t)+g(z, \bar{z})
$$

where

$$
\begin{aligned}
g(z, \bar{z}) & =\bar{q}^{*}(0) F(0, W(z, \bar{z}, 0)+2 \operatorname{Re}[z(t) q(0)]) \\
& =g_{20} \frac{z^{2}}{2}+g_{11} z \bar{z}+g_{02} \frac{\bar{z}^{2}}{2}+g_{21} \frac{z^{2} \bar{z}}{2}+\cdots .
\end{aligned}
$$

It follows from (36) and (43) that

$$
\begin{aligned}
\dot{W} & =\dot{x}_{t}-\dot{z} q-\dot{\bar{z}} \bar{q} \\
& = \begin{cases}A(0) W-2 \operatorname{Re}\left[\bar{q}^{*}(0) F_{0} q(\vartheta)\right], & \vartheta \in\left[-\tau_{1}, 0\right), \\
A(0) W-2 \operatorname{Re}\left[\bar{q}^{*}(0) F_{0} q(0)\right]+F_{0}, & \vartheta=0,\end{cases} \\
& =A W+H(z, \bar{z}, \vartheta),
\end{aligned}
$$

where

$$
\begin{aligned}
H(z, \bar{z}, \vartheta)= & \operatorname{Re}[g(z, \bar{z}) q(0)] \\
& +F(0, W(z, \bar{z}, \vartheta)+2 \operatorname{Re}[z(t) q(\vartheta)]) \\
= & H_{20}(\vartheta) \frac{z^{2}}{2}+H_{11}(\vartheta) z \bar{z}+H_{02}(\vartheta) \frac{\bar{z}^{2}}{2}+\cdots .
\end{aligned}
$$

Expanding the above series and comparing the coefficients, we get

$$
\begin{gathered}
{\left[A(0)-2 i \omega_{0}\right] W_{20}(\vartheta)=-H_{20}(\vartheta),} \\
A(0) W_{11}(\vartheta)=-H_{11}(\vartheta), \\
{\left[A(0)+2 i \omega_{0}\right] W_{02}(\vartheta)=-H_{02}(\vartheta) .}
\end{gathered}
$$

Now $q^{*}(0)=B$ implies

$$
g(z, \bar{z})=\bar{B} \frac{s f^{\prime \prime}\left(k_{*}\right)}{2} x_{t-\tau_{1}}^{2}+\bar{B} \frac{s f^{\prime \prime \prime}\left(k_{*}\right)}{3 !} x_{t-\tau_{1}}^{3}+\cdots .
$$

Noticing that $x_{t}(\vartheta)=W(z, \bar{z}, \vartheta)+z q(\vartheta)+\bar{z} \bar{q}(\vartheta)$, we derive

$$
\begin{aligned}
x_{t-\tau_{1}}= & W_{20}\left(-\tau_{1}\right) \frac{z^{2}}{2}+W_{11}\left(-\tau_{1}\right) z \bar{z} \\
& +W_{02}\left(-\tau_{1}\right) \frac{\bar{z}^{2}}{2}+\cdots+e^{-i \omega_{0} \tau_{1}} z(t)+e^{i \omega_{0} \tau_{1}} \bar{z}(t) .
\end{aligned}
$$

Then substituting this into (50) and comparing the coefficients with (46), the following hold:

$$
\begin{gathered}
g_{20}=\bar{B} s f^{\prime \prime}\left(k_{*}\right) e^{-2 i \omega_{0} \tau_{1}}, \\
g_{11}=\bar{B} s f^{\prime \prime}\left(k_{*}\right), \\
g_{02}=\bar{B} s f^{\prime \prime}\left(k_{*}\right) e^{2 i \omega_{0} \tau_{1}}, \\
g_{21}=\bar{B}\left\{s f^{\prime \prime}\left(k_{*}\right)\left[2 W_{11}\left(-\tau_{1}\right) e^{-i \omega_{0} \tau_{1}}+W_{20}\left(-\tau_{1}\right) e^{i \omega_{0} \tau_{1}}\right]\right. \\
\left.+s f^{\prime \prime \prime}\left(k_{*}\right) e^{-i \omega_{0} \tau_{1}}\right\} .
\end{gathered}
$$

In order to compute $g_{21}$, we need to know $W_{20}(\vartheta)$ and $W_{11}(\vartheta)$. For $\vartheta \in\left[-\tau_{1}, 0\right]$, we have

$$
\begin{aligned}
H(z, \bar{z}, \theta)= & -2 \operatorname{Re}\left\{\bar{q}^{*}(0) F_{0} q(\theta)\right\}=-g q(\theta)-\bar{g} \bar{q}(\theta) \\
= & -\left(g_{20} \frac{z^{2}}{2}+g_{11} z \bar{z}+g_{02} \frac{\bar{z}^{2}}{2}+\cdots\right) q(\theta) \\
& -\left(\bar{g}_{20} \frac{\bar{z}^{2}}{2}+\bar{g}_{11} \bar{z} z+\bar{g}_{02} \frac{z^{2}}{2}+\cdots\right) \bar{q}(\theta) .
\end{aligned}
$$

Comparing the coefficients with those in (48) yields

$$
\begin{aligned}
& H_{20}(\theta)=-g_{20} q(\theta)-\bar{g}_{02} \bar{q}(\theta), \\
& H_{11}(\theta)=-g_{11} q(\theta)-\bar{g}_{11} \bar{q}(\theta) .
\end{aligned}
$$


From (49)

$$
\begin{gathered}
\dot{W}_{20}(\theta)=2 i \omega_{0} W_{20}(\theta)+g_{20} q(\theta)+\bar{g}_{02} \bar{q}(\theta) \\
\dot{W}_{11}(\theta)=g_{11} q(\theta)+\bar{g}_{11} \bar{q}(\theta) .
\end{gathered}
$$

Solving $W_{20}(\theta)$, we have

$$
W_{20}(\theta)=-\frac{g_{20}}{i \omega_{0}} e^{i \omega_{0} \theta}-\frac{\bar{g}_{02}}{3 i \omega_{0}} e^{-i \omega_{0} \theta}+E_{1} e^{2 i \omega_{0} \theta},
$$

and, similarly for $W_{11}(\theta)$,

$$
W_{11}(\theta)=\frac{g_{11}}{i \omega_{0}} e^{i \omega_{0} \theta}-\frac{\bar{g}_{11}}{i \omega_{0}} e^{-i \omega_{0} \theta}+E_{2} .
$$

Here $E_{1}$ and $E_{2}$ are constants to be determined by setting $\theta=$ 0 in $H(z, \bar{z}, \theta)$. A direct computation shows

$$
\begin{gathered}
E_{1}=\frac{s f^{\prime \prime}\left(k_{*}\right) e^{-2 i \omega_{0} \tau_{1}}}{2 i \omega_{0}+\left[s f^{\prime}\left(k_{*}\right)-\delta\right] e^{-2 i \omega_{0} \tau_{1}}+\delta e^{-2 i \omega_{0} \tau_{2}^{0}}}, \\
E_{2}=\frac{B}{s f^{\prime}\left(k_{*}\right)} .
\end{gathered}
$$

Then all $g_{i j}$ have been obtained, and thus we can compute the quantities

$$
\begin{gathered}
c_{1}(0)=\frac{i}{2 \omega_{0}}\left(g_{11} g_{20}-2\left|g_{11}\right|^{2}-\frac{\left|g_{02}\right|^{2}}{3}\right)+\frac{g_{21}}{2}, \\
\mu_{2}=-\frac{\operatorname{Re}\left[c_{1}(0)\right]}{\operatorname{Re}\left[\lambda^{\prime}\left(\tau_{2}^{0}\right)\right]} \\
\beta_{2}=2 \operatorname{Re}\left[c_{1}(0)\right] \\
T_{2}=-\frac{\operatorname{Im}\left[c_{1}(0)\right]+\mu_{2} \operatorname{Im}\left[\lambda^{\prime}\left(\tau_{2}^{0}\right)\right]}{\omega_{0}},
\end{gathered}
$$

which determine the properties of bifurcating periodic solutions at the critical value $\tau_{2}^{0}$. From the discussion above, we have the following result.

Theorem 9. Let $k_{*}$ be the unique positive equilibrium of the model (2). Then one has the following.

(1) $\mu_{2}$ determines the direction of the Hopf bifurcation when $\tau=\tau_{2}^{0}$ : if $\mu_{2}>0$ (resp., $\mu_{2}<0$ ), then the Hopf bifurcation is supercritical (resp., subcritical) and the bifurcating periodic solution exists for $\tau>\tau_{2}^{0}$ (resp., $\tau<\tau_{2}^{0}$ ) in a sufficiently small $\tau_{2}^{0}$-neighbourhood.

(2) $\beta_{2}$ determines the stability of the bifurcating periodic solution: if $\beta_{2}<0$ (resp., $\beta_{2}>0$ ) the bifurcating periodic solution is locally asymptotically stable (resp., unstable).

(3) $T_{2}$ determines the period of the bifurcating periodic solution: if $T_{2}>0$ (resp., $T_{2}<0$ ) the period increases (resp., decreases).

\section{Numerical Simulations}

This section is concerned with some numerical simulations of the mathematical model (2) with the aim of exploring the analytical results. The model is characterized by four nonnegative parameters $\left(s, \delta, \tau_{1}, \tau_{2}\right)$ and the function $f$. In what follows we restrict our attention to the Cobb-Douglas function; this function reads

$$
f(k(t))=A[k(t)]^{\alpha},
$$

with $\alpha \in(0,1)$ and $A>0$.

The first set of simulations refers to the following case:

$$
\begin{array}{ccc}
s=0.11, & \delta=0.8, & \alpha=0.1, \\
A=1, & \tau_{1}=3, & \tau_{2}=2,
\end{array}
$$

where $\tau_{1}>\tau_{2}$. Figure 1(a) shows that the function $k(t)$ reaches the stationary state (the equilibrium $k_{*}$ ). This equilibrium is stable; see Figure 1(b), where the evolution of $k^{\prime}(t)$ versus $k(t)$ is depicted for $t \in[0,100]$.

The dynamics depicted by Figure 1 does not change from the qualitative viewpoint when $\alpha \in[0.02,1)$. Indeed when the parameter $\alpha$ varies in the interval $[00.2,1)$ the time necessary for reaching the stationary state increases but the behavior is that of Figure 1. When $\alpha<0.2$ the time evolution of $k(t)$ is shown in Figure 2. In this simulation the time length has been increased in order to better visualize the evolution. As Figure 2(b) shows, the equilibrium is now instable.

The second set of simulations refers to the following case:

$$
\begin{array}{ccc}
s=0.11, & \delta=0.8, & \alpha=0.1, \\
A=1, & \tau_{1}=1, & \tau_{2}=2 .
\end{array}
$$

These simulations take into account the case $\tau_{1}<\tau_{2}$. As Figure 3 shows, oscillations occur for a long time (about $t \in$ $[0,1500])$, with respect to the previous case, before reaching the equilibrium. In this case the equilibrium is stable but a very long time is necessary to reach it; see Figure 3(b). It is sufficient to increase $\alpha$ for obtaining the stationary state rapidly. These simulations suggest that when the value of $\alpha$ increases then the time necessary to reach the stationary state decreases. Moreover, if the difference $\tau_{2}-\tau_{1}>0$ increases, then the stability of the equilibrium is lost for all $\alpha \in(0.1)$.

Finally, we would show some numerical simulations related to the evolution of $k^{\prime}(t)$ versus $k(t)$. Figure 4 shows the instability of the equilibrium for $s=0.41, \delta=0.35, \alpha=0.8$, $A=1, \tau_{1}=10, \tau_{2}=2$, see Figure 4(a); it is worth stressing that the equilibrium is reached for $t \geq 3500$. In this case, when we increase the magnitude of $s$ we are able to reach the equilibrium more rapidly, see Figure 4(b), which is obtained for $s=0.91$ and $t \in[0,3500]$. The equilibrium is rapidly reached if we also decrease the magnitude of $\alpha$.

\section{Conclusions and Research Perspectives}

In the present paper, a generalization of the Solow model by inserting two time delays has been considered. The delays, respectively, represent the time employed in order that 


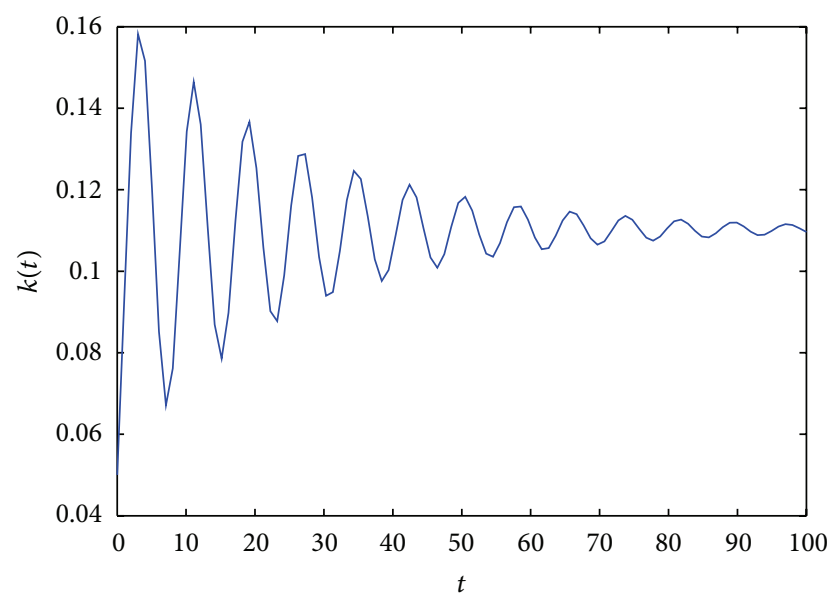

(a)

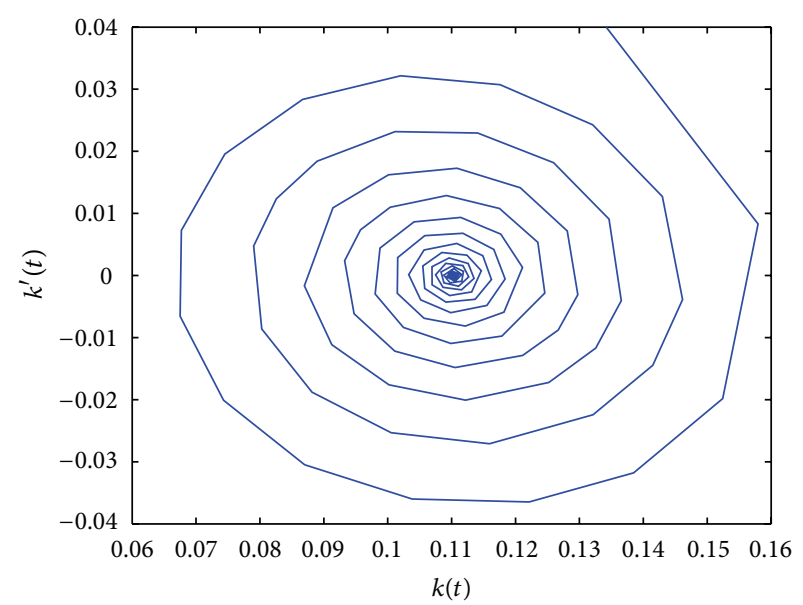

(b)

Figure 1: The time evolution of the function $k(t)$ for $s=0.11, \delta=0.8, \alpha=0.1, A=1, \tau_{1}=3$, and $\tau_{2}=2$ (a). The $k^{\prime}(t)$ versus $k(t)$, for $t \in[0,100](\mathrm{b})$.

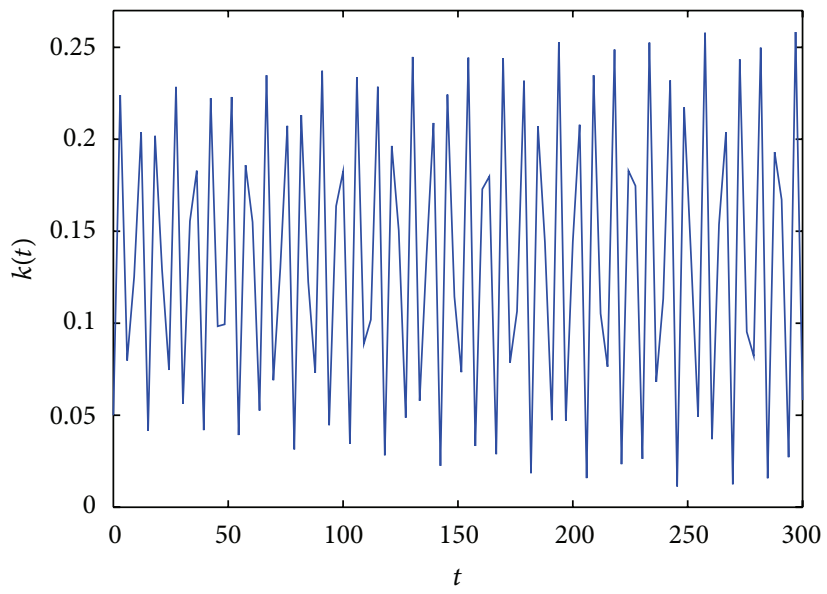

(a)

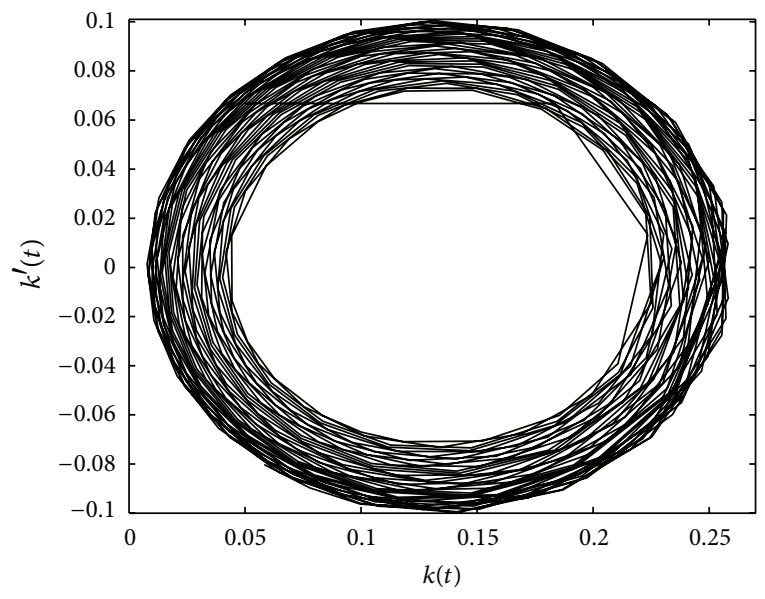

(b)

Figure 2: The time evolution of the function $k(t)$ for $s=0.11, \delta=0.8, \alpha=0.01, A=1, \tau_{1}=3$, and $\tau_{2}=2$ (a). The $k^{\prime}(t)$ versus $k(t)$, for $t \in[0,300](\mathrm{b})$.

the capital is used for production and the necessary time so that the capital is depreciated. Specifically, an asymptotic analysis has been performed referring to the stability analysis of the steady state and the conditions under which a Hopf bifurcation appears.

According to the analysis developed in this paper, the stability of the positive equilibrium changes as the time delays vary. Indeed if $\tau_{2}=0$, the positive equilibrium is always locally asymptotically stable; if $\tau_{1}=0$ the positive equilibrium can be locally asymptotically stable or unstable and a Hopf bifurcation occurs; the dynamics is more complicated when the two time delays are both different from zero (see Theorem 8 where as the reader can see the investigation of stability switches becomes quite complicated). This shows that the time delays play an important role in the dynamics of the model. Then, based on the analysis of the existence of the Hopf bifurcation, by using the center manifold theory and the normal form method, an explicit algorithm for determining the direction of the Hopf bifurcation and the stability of the bifurcating periodic solutions has been derived. This means that one can obtain the important quantities which determine the properties of bifurcating periodic solutions at the critical value; see Theorem 9. According to our results, we can say that the model with two independent time delays has much more complicated dynamics than the model with only one time delay. That is why it seems to be more realistic.

The introduction of time delays can be also performed in the mathematical model developed in [36] for the mammary carcinoma. Indeed the stability analysis developed in the present paper can help to reach more results in the cancerimmune system competition. From a biological point of view, the Hopf bifurcation means that for small values of parameters the nontrivial stationary solution to the model in [36] is stable, and we do not observe radical changes in the competition. Otherwise, nontrivial stationary solution can oscillate and the amplitude of the oscillations about 


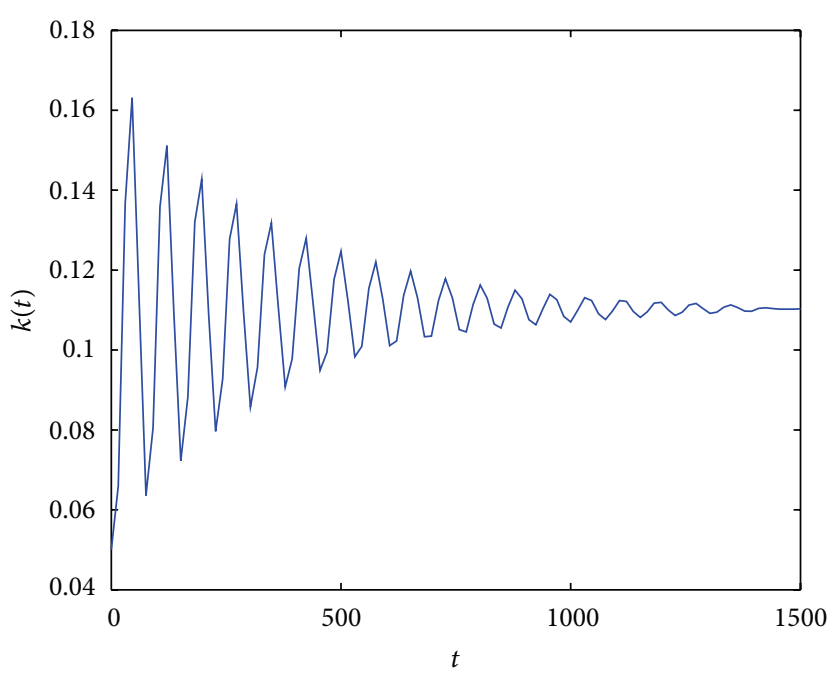

(a)

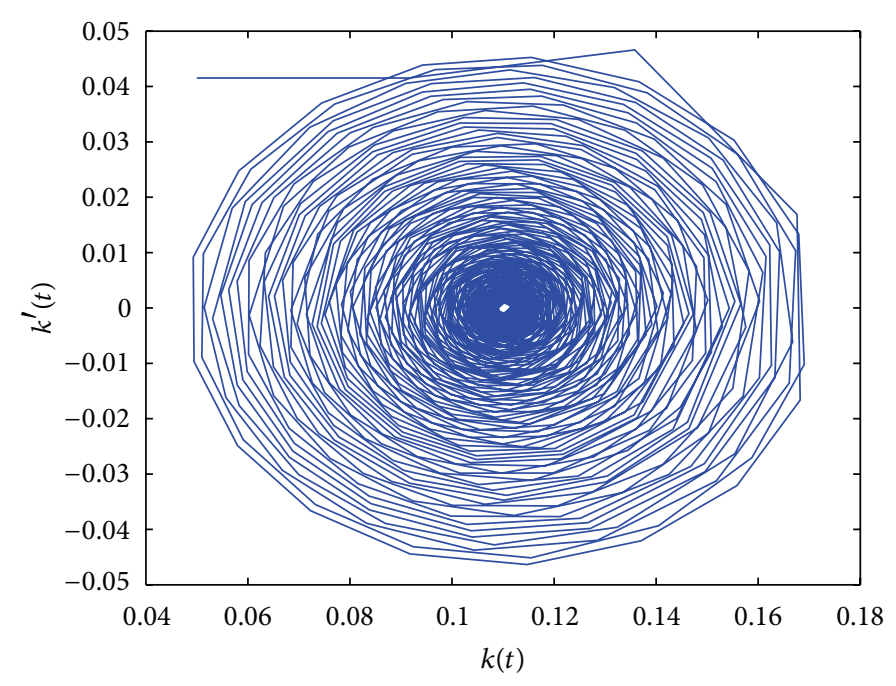

(b)

Figure 3: The time evolution of the function $k(t)$ for $s=0.11, \delta=0.8, \alpha=0.1, A=1, \tau_{1}=1$, and $\tau_{2}=2$ (a). The $k^{\prime}(t)$ versus $k(t)$, for $t \in[0,1300](b)$.

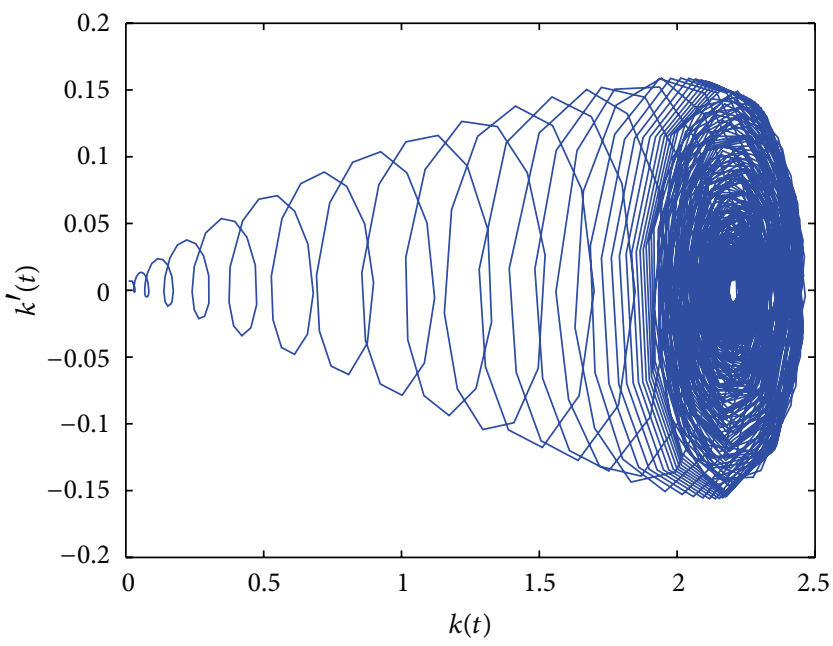

(a)

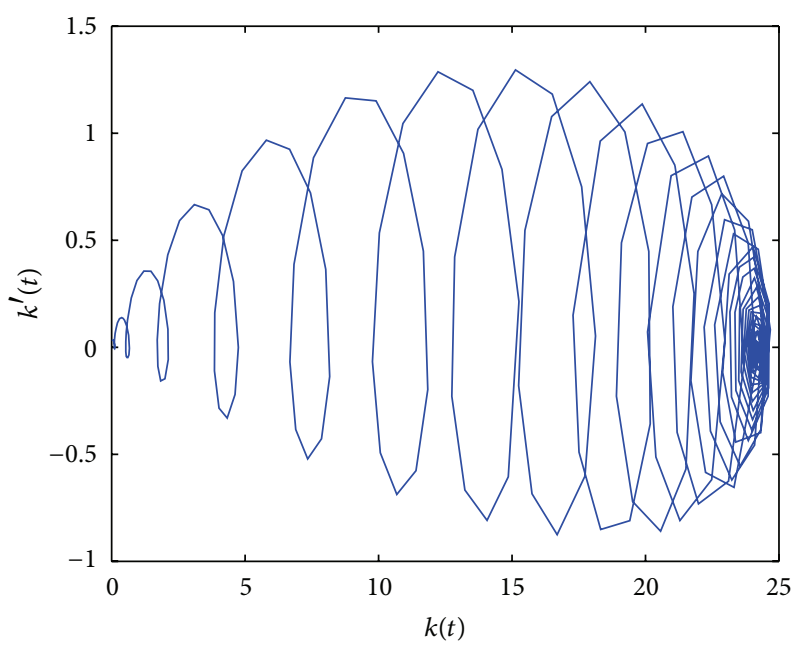

(b)

Figure 4: The time evolution of $k^{\prime}(t)$ versus $k(t)$ for $s=0.41, \delta=0.35, \alpha=0.8, A=1, \tau_{1}=10, \tau_{2}=2$, and $t \in[0,3500]$ (a). The time evolution of $k^{\prime}(t)$ versus $k(t)$ for $s=0.91, \delta=0.35, \alpha=0.8, A=1, \tau_{1}=10, \tau_{2}=2$, and $t \in[0,3500](\mathrm{b})$.

the stationary solution remains constant. This case simply corresponds to the situation when the competition oscillates in time.

The Hopf bifurcation analysis developed in this paper must be revised if the mathematical models are not based on ordinary differential equations. Recently an increasing number of partial differential equation models for tumor growth or therapy have been developed; see the references section of paper [12] and the references cited in the recent review paper [37].

Moreover thermostated integrodifferential equations have been proposed in papers [38-43] for the modeling of biological systems, vehicular traffic, crowd and swarm dynamics, and economic systems subjected to external force fields. The introduction of the Gaussian isokinetic thermostat ensures the reaching of stationary states whose existence has been proved in [44]. The introduction of multiple time delays in thermostated equations, their stability, and bifurcation analysis is a future research perspective.

It is worth stressing that also the Boltzmann equation with the one-dimensional Bhatnagar-Gross-Krook relaxation type operator [45] and the Kac equation have been coupled with a Gaussian isokinetic thermostat; the existence of stationary solutions is ensured also within these frameworks; see papers [46-48].

\section{Conflict of Interests}

The authors declare that there is no conflict of interests regarding the publication of this paper. 


\section{Acknowledgments}

The first author acknowledges the support by the FIRB project RBID08PP3J-Metodi matematici e relativi strumenti per la modellizzazione e la simulazione della formazione di tumori, competizione con il sistema immunitario, e conseguenti suggerimenti terapeutici. The authors acknowledge the financial support of MEDAlics-Research Center for Mediterranean Relations.

\section{References}

[1] T. Erneux, Applied Delay Differential Equations, Springer, New York, NY, USA, 2009.

[2] P. J. Cunningham and W. J. Wangersky, Time Lag in Population Models, Yale, 1958.

[3] G. I. Marchuk, Mathematical Modelling of Immune Response in Infectious Diseases, Kluwer Academic, Dordrecht, Germany, 1997.

[4] U. Foryś, "Interleukin mathematical model of an immune system," Journal of Biological Systems, vol. 3, pp. 889-902, 1995.

[5] U. Foryś, "Global analysis of Marchuks model in case of strong immune system," Journal of Biological Systems, vol. 8, pp. 331346, 2000.

[6] M. Bodnar and U. Foryś, "A model of immune system with time-dependent immune reactivity," Nonlinear Analysis: Theory, Methods \& Applications, vol. 70, no. 2, pp. 1049-1058, 2009.

[7] U. Foryś and M. Bodnar, “Time delays in proliferation process for solid avascular tumour," Mathematical and Computer Modelling, vol. 37, no. 11, pp. 1201-1209, 2003.

[8] H. M. Byrne, "The effect of time delays on the dynamics of avascular tumor growth," Mathematical Biosciences, vol. 144, no. 2, pp. 83-117, 1997.

[9] M. J. Piotrowska, "Hopf bifurcation in a solid avascular tumour growth model with two discrete delays," Mathematical and Computer Modelling, vol. 47, no. 5-6, pp. 597-603, 2008.

[10] M. Bodnar and U. Foryś, “Three types of simple DDE's describing tumour growth," Journal of Biological Systems, vol. 15, pp. 1-19, 2007.

[11] M. Bodnar and U. Foryś, "Global stability and the Hopf bifurcation for some class of delay differential equation," Mathematical Methods in the Applied Sciences, vol. 31, no. 10, pp. 1197-1207, 2008.

[12] B. Shi, F. Zhang, and S. Xu, "Hopf bifurcation of a mathematical model for growth of tumors with an action of inhibitor and two time delays," Abstract and Applied Analysis, vol. 2011, Article ID 980686, 10 pages, 2011.

[13] N. Bielczyk, U. Foryś, and T. Platkowski, "Dynamical models of Dyadic interactions with delay," The Journal of Mathematical Sociology, vol. 37, no. 4, 2013.

[14] S. Invernizzi and A. Medio, "On lags and chaos in economic dynamic models," Journal of Mathematical Economics, vol. 20, no. 6, pp. 521-550, 1991.

[15] P. J. Zak, "Kaleckian lags in general equilibrium," Review of Political Economy, vol. 11, no. 3, pp. 321-330, 1999.

[16] G. Dibeh, "Speculative dynamics in a time-delay model of asset prices," Physica A, vol. 355, no. 1, pp. 199-208, 2005.

[17] M. Szydlowski, A. Krawiec, and J. Tobola, "Nonlinear oscillations in business cycle model with time lags," Chaos, Solitons and Fractals, vol. 12, no. 3, pp. 505-517, 2001.
[18] L. Zhou and Y. Li, "A dynamic IS-LM business cycle model with two time delays in capital accumulation equation," Journal of Computational and Applied Mathematics, vol. 228, no. 1, pp. 182$187,2009$.

[19] C. Bianca and L. Guerrini, "On the Dalgaard-Strulik model with logistic population growth rate and delayed-carrying capacity," Acta Applicandae Mathematicae, vol. 128, pp. 39-48, 2013.

[20] C. Bianca, M. Ferrara, and L. Guerrini, "Hopf bifurcations in a delayed-energy-based model of capital accumulation," Applied Mathematics \& Information Sciences, vol. 7, no. 1, pp. 139-143, 2013.

[21] C. Bianca, M. Ferrara, and L. Guerrini, "The Cai model with time delay: existence of periodic solutions and asymptotic analysis," Applied Mathematics \& Information Sciences, vol. 7, no. 1, pp. 21-27, 2013.

[22] Y. Ma, "Global Hopf bifurcation in the Leslie-Gower predatorprey model with two delays," Nonlinear Analysis: Real World Applications, vol. 13, no. 1, pp. 370-375, 2012.

[23] Y. Song, J. Wei, and M. Han, "Local and global Hopf bifurcation in a delayed hematopoiesis model," International Journal of Bifurcation and Chaos in Applied Sciences and Engineering, vol. 14, no. 11, pp. 3909-3919, 2004.

[24] J. Wei, "Bifurcation analysis in a scalar delay differential equation," Nonlinearity, vol. 20, no. 11, pp. 2483-2498, 2007.

[25] E. Burger, “On the stability of certain economic systems," Econometrica, vol. 24, pp. 488-493, 1956.

[26] N. D. Hayes, "Roots of the transcendental equation associated with a certain difference-differential equation," Journal of the London Mathematical Society, vol. 25, pp. 226-232, 1950.

[27] J. K. Hale and W. Z. Huang, "Global geometry of the stable regions for two delay differential equations," Journal of Mathematical Analysis and Applications, vol. 178, no. 2, pp. 344-362, 1993.

[28] X. Li, S. Ruan, and J. Wei, "Stability and bifurcation in delaydifferential equations with two delays," Journal of Mathematical Analysis and Applications, vol. 236, no. 2, pp. 254-280, 1999.

[29] J. Wei and Y. Yuan, "Synchronized Hopf bifurcation analysis in a neural network model with delays," Journal of Mathematical Analysis and Applications, vol. 312, no. 1, pp. 205-229, 2005.

[30] J. K. Hale and W. Z. Huang, "Global geometry of the stable regions for two delay differential equations," Journal of Mathematical Analysis and Applications, vol. 178, no. 2, pp. 344-362, 1993.

[31] M. J. Piotrowska, "A remark on the ODE with two discrete delays," Journal of Mathematical Analysis and Applications, vol. 329, no. 1, pp. 664-676, 2007.

[32] R. M. Solow, "A contribution to the theory of economic growth," Quarterly Journal of Economics, vol. 70, pp. 65-94, 1956.

[33] S. Ruan and J. Wei, "On the zeros of transcendental functions with applications to stability of delay differential equations with two delays," Dynamics of Continuous, Discrete \& Impulsive Systems A, vol. 10, no. 6, pp. 863-874, 2003.

[34] J. K. Hale and S. M. Verduyn Lunel, Introduction to FunctionalDifferential Equations, Springer, New York, NY, USA, 1993.

[35] B. D. Hassard, N. D. Kazarinoff, and Y. H. Wan, Theory and Applications of Hopf Bifurcation, Cambridge University Press, 1981.

[36] C. Bianca and M. Pennisi, "The triplex vaccine effects in mammary carcinoma: a nonlinear model in tune with SimTriplex," Nonlinear Analysis: Real World Applications, vol. 13, no. 4, pp. 1913-1940, 2012. 
[37] C. Bianca, "Thermostatted kinetic equations as models for complex systems in physicsand life sciences," Physics of Life Reviews, vol. 9, pp. 359-399, 2012.

[38] C. Bianca, "Kinetic theory for active particles modelling coupled to Gaussian thermostats," Applied Mathematical Sciences, vol. 6, no. 13-16, pp. 651-660, 2012.

[39] C. Bianca, "Modeling complex systems by functional subsystems representation and thermostatted-KTAP methods," Applied Mathematics \& Information Sciences, vol. 6, pp. 495-499, 2012.

[40] C. Bianca, "An existence and uniqueness theorem to the Cauchy problem for thermostatted-KTAP models," International Journal of Mathematical Analysis, vol. 6, no. 17-20, pp. 813-824, 2012.

[41] C. Bianca, M. Ferrara, and L. Guerrini, "High-order moments conservation in thermostatted kinetic models," Journal of Global Optimization, 2013.

[42] C. Bianca, "Onset of nonlinearity in thermostatted active particles models for complex systems," Nonlinear Analysis: Real World Applications, vol. 13, no. 6, pp. 2593-2608, 2012.

[43] C. Bianca, "Controllability in hybrid kinetic equations modeling nonequilibrium multicellular systems," The Scientific World Journal, vol. 2013, Article ID 274719, 6 pages, 2013.

[44] C. Bianca, "Existence of stationary solutions in kinetic models with Gaussian thermostats," Mathematical Methods in the Applied Sciences, vol. 36, pp. 1768-1775, 2013.

[45] P. Degond and B. Wennberg, "Mass and energy balance laws derived from high-field limits of thermostatted Boltzmann equations," Communications in Mathematical Sciences, vol. 5, no. 2, pp. 355-382, 2007.

[46] V. Bagland, B. Wennberg, and Y. Wondmagegne, "Stationary states for the noncutoff Kac equation with a Gaussian thermostat," Nonlinearity, vol. 20, no. 3, pp. 583-604, 2007.

[47] B. Wennberg and Y. Wondmagegne, "Stationary states for the Kac equation with a Gaussian thermostat," Nonlinearity, vol. 17, no. 2, pp. 633-648, 2004.

[48] B. Wennberg and Y. Wondmagegne, "The Kac equation with a thermostatted force field," Journal of Statistical Physics, vol. 124, no. 2-4, pp. 859-880, 2006. 


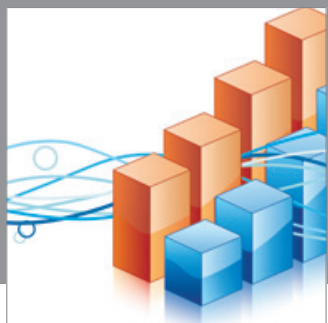

Advances in

Operations Research

mansans

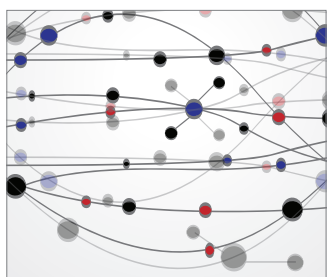

The Scientific World Journal
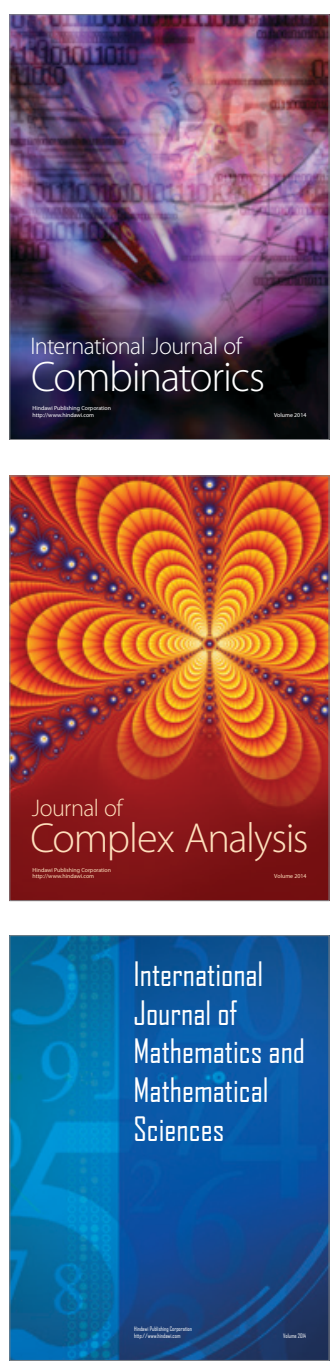
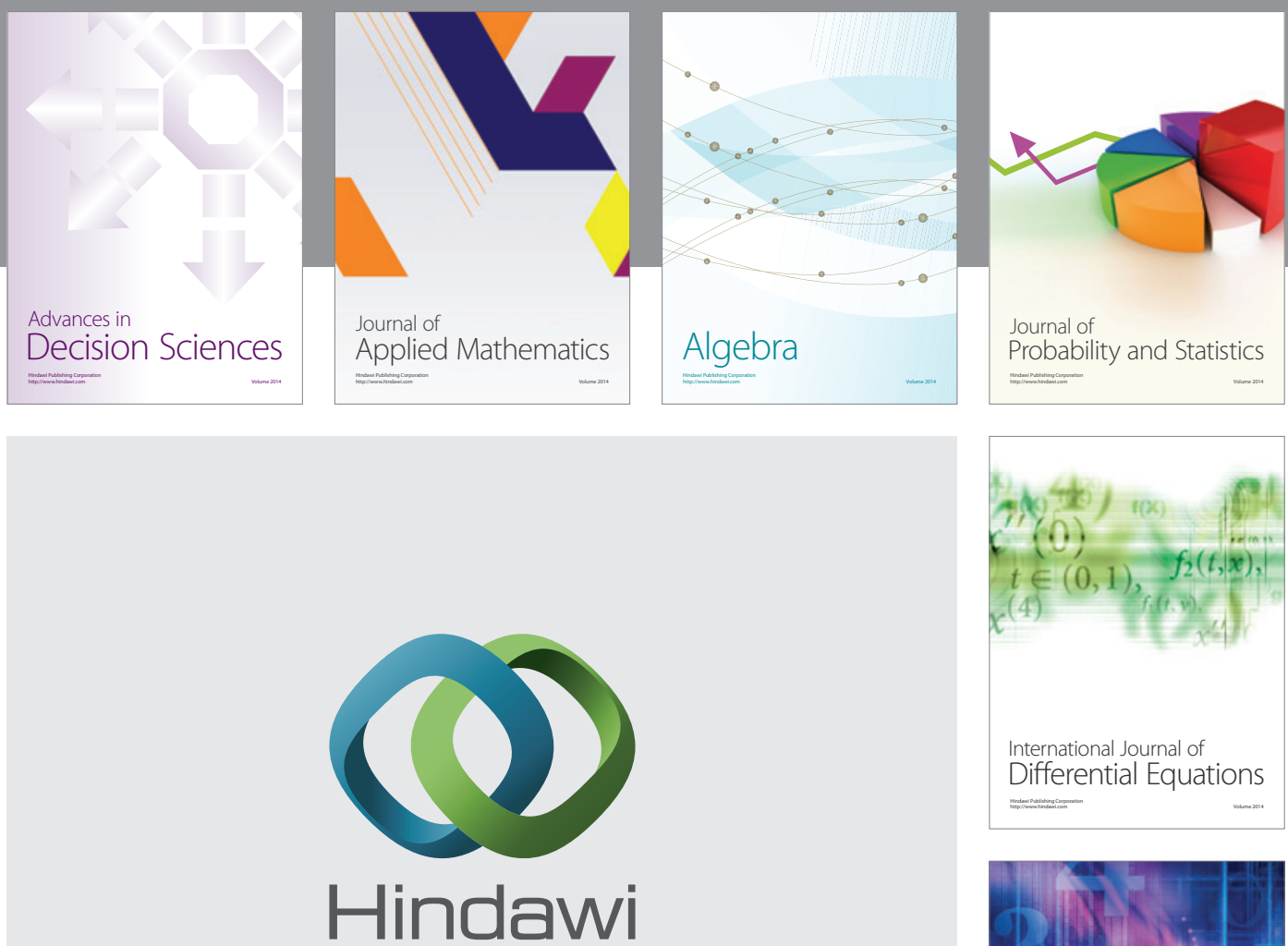

Submit your manuscripts at http://www.hindawi.com
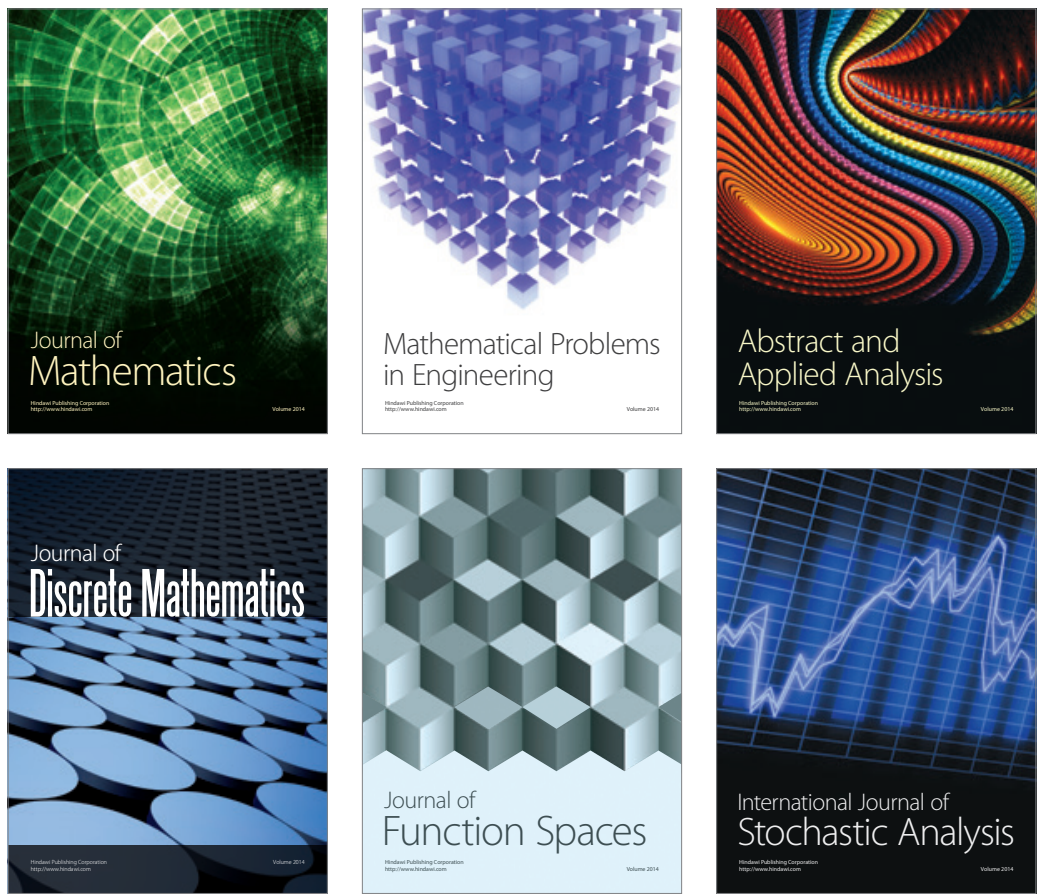

Journal of

Function Spaces

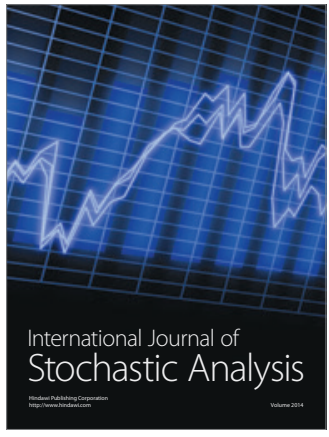

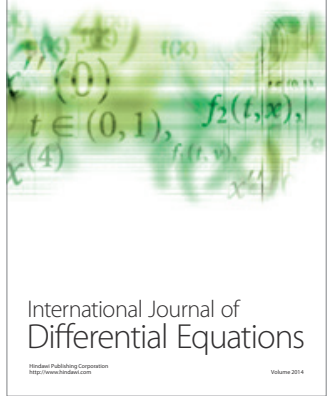
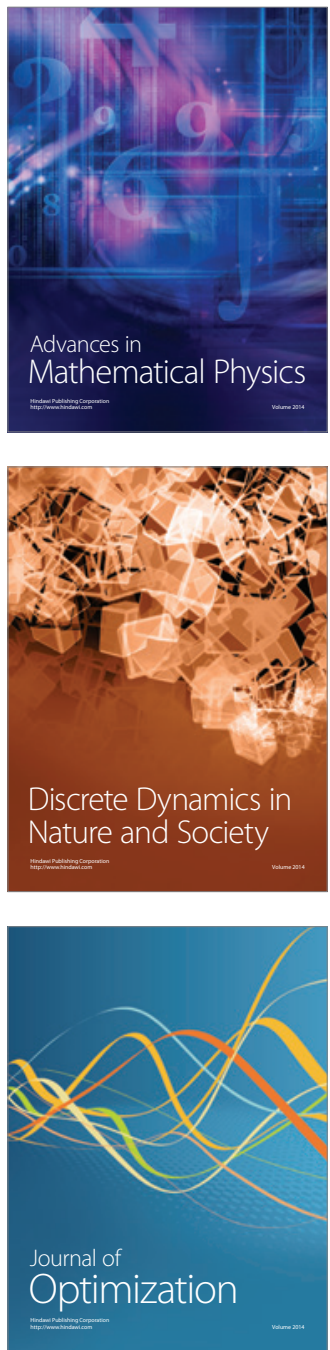